\title{
Diels-Alder Reaction for Tumor Pretargeting: In Vivo Chemistry Can Boost Tumor Radiation Dose Compared with Directly Labeled Antibody
}

\author{
Raffaella Rossin ${ }^{1}$, Tilman Läppchen ${ }^{1}$, Sandra M. van den Bosch ${ }^{1}$, Richard Laforest ${ }^{2}$, and Marc S. Robillard ${ }^{1}$ \\ ${ }^{I}$ Department of Minimally Invasive Healthcare, Philips Research, Eindhoven, The Netherlands; and ${ }^{2}$ Division of Radiological \\ Sciences, Washington University School of Medicine, St. Louis, Missouri
}

Current pretargeting systems use noncovalent biologic interactions, which are prone to immunogenicity. We previously developed a novel approach based on the bioorthogonal reaction between a radiolabeled tetrazine and an antibody-conjugated trans-cyclooctene (TCO). However, the tumor-to-blood ratio was low due to reaction with freely circulating antibody-TCO. Methods: Here we developed 2 tetrazine-functionalized clearing agents that enable rapid reaction with and removal of a TCO-tagged antibody (CC49) from blood. Next, we incorporated this approach into an optimized pretargeting protocol in LS174T-bearing mice. Then we compared the pretargeted ${ }^{177} \mathrm{Lu}$-labeled tetrazine with ${ }^{177} \mathrm{Lu}$-labeled CC49. The biodistribution data were used for mouse and human dosimetry calculations. Results: The use of a clearing agent led to a doubling of the tetrazine tumor uptake and a 125 -fold improvement of the tumor-to-blood ratio at $3 \mathrm{~h}$ after tetrazine injection. Mouse dosimetry suggested that this should allow for an 8-fold higher tumor dose than is possible with nonpretargeted radioimmunotherapy. Also, humans treated with CC49-TCO-pretargeted ${ }^{177} \mathrm{Lu}$-tetrazine would receive a dose to nontarget tissues 1 to 2 orders of magnitude lower than with directly labeled CC49. Conclusion: The in vivo performance of chemical pretargeting falls within the range of results obtained for the clinically validated pretargeting approaches in mice, with the advantage of potentially allowing for fractionated radiotherapy as a result of a lower likelihood of immunogenicity. These findings demonstrate that biologic pretargeting concepts can be translated to rapid bioorthogonal chemical approaches with retained potential.

Key Words: Diels-Alder; pretargeting; clearing agent; ${ }^{177} \mathrm{Lu}$; dosimetry

J Nucl Med 2013; 54:1989-1995

DOI: 10.2967/jnumed.113.123745

$\mathbf{T}$ he use of radiolabeled monoclonal antibodies (mAbs) for radioimmunotherapy of cancer is hampered by low tumor-to-nontumor ratios resulting in dose-limiting side effects in the bone marrow and in low tumor doses in patients (1). Pretargeting capitalizes on

Received Mar. 26, 2013; revision accepted Jul. 3, 2013.

For correspondence or reprints contact: Marc S. Robillard, Tagworks Pharmaceuticals, High Tech Campus 11, 5656 AE, Eindhoven, The Netherlands.

E-mail: marc.robillard@tagworkspharma.com

Published online Oct. 3, 2013.

COPYRIGHT (C 2013 by the Society of Nuclear Medicine and Molecular Imaging, Inc. the tumor-seeking capabilities of long-circulating mAbs and on the fast distribution and clearance of small molecules with the aim of enabling the treatment of solid tumors in particular (2). Several pretargeting approaches have been tested in vivo (2-5), and to date, 2 have been evaluated in the clinic (2): systems that capture the probe using the extremely high affinity of the biotin-streptavidin interaction (association constant, $\sim 10^{15} \mathrm{M}^{-1}$ ) and those that use antibody-hapten interactions (association constant, $\sim 10^{8}-10^{11} \mathrm{M}^{-1}$ ). However, these biologic pretargeting systems, especially streptavidin constructs, are prone to immunogenicity, thus precluding repeated treatment cycles, which have proven critical to maximize the efficacy for other treatment modalities such as external-beam radiotherapy and chemotherapy. Furthermore, many of these systems require extensive reengineering and perturbation of the parent $\mathrm{mAb}$.

Bioorthogonal chemical reactions ( 6 ) may be an alternative to biologic pretargeting interactions for the recruitment of the small radiolabeled probe to the tumor-bound $\mathrm{mAb}$ (Fig. 1). Such a chemical approach is less likely to give rise to immunogenicity and may in addition enable universal and straightforward tagging and in vivo tracking of mAbs without severe perturbation of the in vivo properties of the parent construct. Although the use of organic chemistry in living beings is a highly sought-after approach, only the inverse-electron-demand Diels-Alder reaction between strained trans-cyclooctene (TCO) and electron-deficient tetrazines has so far demonstrated sufficient potential for such demanding conditions $(6-10)$. We reported previously that the use of this reaction could indeed be extended to a living system, showing pronounced and specific localization of an ${ }^{111}$ In-labeled DOTAtetrazine probe in LS174T-tumored mice that were pretargeted with anti-TAG72 mAb CC49-TCO (8). However, the tumor-toblood (T/B) ratio was still low because of the reaction between ${ }^{111}$ In-tetrazine and freely circulating CC49-TCO. Recently, the groups of Weissleder and Lewis achieved similar results with, respectively, an ${ }^{18} \mathrm{~F}$ - and ${ }^{64} \mathrm{Cu}$-labeled tetrazine $(9,10)$. So far the inverse-electron-demand Diels-Alder approach has not led to an improvement of T/B ratio compared with directly labeled $\mathrm{mAb}$. Ideally, the probe should be administered when the mAb has cleared completely from circulation. Since in humans intact mAbs take weeks to clear, requiring an unrealistic on-tumor stability of the mAb tag, they can instead be removed by injection of a clearing agent (CA). In the clinic, biotin-functionalized, liver-directed CAs have successfully been used to quickly capture streptavidintagged antibodies, boosting T/B ratios (11).

To extend the concept of organic chemistry in mice to effective pretargeted imaging and radiotherapy with potential for clinical translation, we set out to match the performance of the clinically 


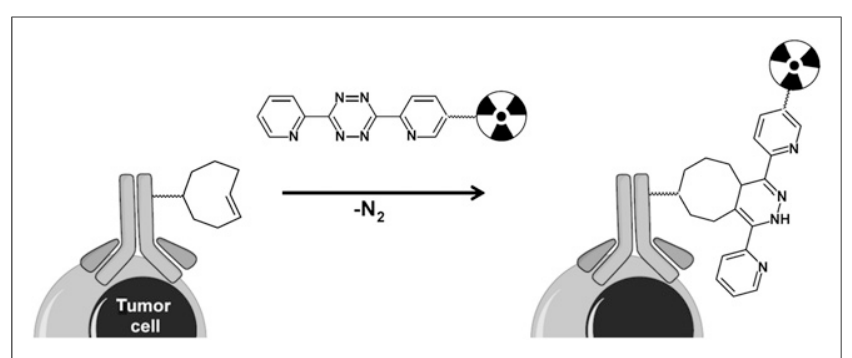

FIGURE 1. Tumor pretargeting with the inverse-electron-demand DielsAlder reaction involving TCO-modified $\mathrm{mAb}$ and radiolabeled tetrazine.

validated biologic pretargeting interactions with fast bioorthogonal organic chemistry. We developed a chemical clearing approach (compounds 3 and $\mathbf{4}$ in Fig. 2) that enables the controlled manipulation and removal of tagged biomolecules from blood, and we incorporated this into an optimized pretargeting protocol with a TCO with increased reactivity and stability (12), which led to a doubling of the tetrazine tumor uptake and a 125-fold improvement of the T/B ratio at $3 \mathrm{~h}$ after injection. We used a tetrazine labeled with ${ }^{177} \mathrm{Lu}$ (half-life $\left[\mathrm{t}_{1 / 2}\right], 6.7 \mathrm{~d}$ ), which, in addition to $\gamma$, has a $\beta$ emission that can be used for radiotherapy. We then demonstrated in a dosimetry study that the new protocol should allow for an 8-fold higher total tumor dose than is possible with nonpretargeted radioimmunotherapy, which is on a par with the noncovalent pretargeting approaches.

\section{MATERIALS AND METHODS}

\section{Syntheses of Pretargeting Components}

CC49 production, TCO (1) synthesis, and conjugation and the synthesis of DOTA-tetrazine (2) (Fig. 2) have been described elsewhere $(8,12)$. The syntheses of other compounds and more experimental details are reported in the supplemental data, which are available at http://jnm.snmjournals.org.

\section{DOTA-Tetrazine Radiolabeling}

DOTA-tetrazine (2) $(2 \mathrm{mg} / \mathrm{mL}$ in $0.2 \mathrm{M}$ ammonium acetate buffer, $\mathrm{pH}$ 7.0) was mixed with a suitable amount of ${ }^{177} \mathrm{LuCl}_{3}$ or ${ }^{111} \mathrm{InCl}_{3}$ (PerkinElmer) in $0.2 \mathrm{M}$ ammonium acetate $\mathrm{pH} 5.5$ and incubated at $60^{\circ} \mathrm{C}$ for $5 \mathrm{~min}$. The labeling mixture was then mixed with $10 \mathrm{mM}$ diethylenetriaminepentaacetic acid $(5 \mu \mathrm{L})$ and a $20 \mathrm{mg} / \mathrm{mL}$ gentisic acid solution in saline $(\mathrm{pH} 6.5)$ and incubated for 5 min more. The radiochemical yield and purity were assessed by radio-instant thinlayer chromatography and radio-high-performance liquid chromatography, respectively, after which the labeling mixture was diluted with sterile saline for animal experiments. The specific activity of ${ }^{177} \mathrm{Lu}-2$ used for biodistribution and that of ${ }^{111}$ In-2 used for SPECT/CT imaging was $0.07-0.15$ and approximately $6 \mathrm{MBq} / \mathrm{nmol}$, respectively.

\section{CC49-TCO Radiolabeling}

Radioiodination of CC49-TCO was performed with the BoltonHunter method. Briefly, an adequate amount of $\mathrm{Na}^{125} \mathrm{I}$ (PerkinElmer) in $50 \mu \mathrm{L}$ of phosphate-buffered saline was mixed with $1 \mu \mathrm{g}$ of BoltonHunter reagent (SHPP; Pierce Protein Research) and $100 \mu \mathrm{g}$ of chloramine-T. The solution was mixed for $10-20 \mathrm{~s},{ }^{125}$ I-SHPP was extracted into toluene, and the solution was evaporated to dryness under $\mathrm{N}_{2}$. CC49-TCO $(0.1-0.5 \mathrm{mg}$ in $50-250 \mu \mathrm{L}$ of phosphate-buffered saline) was added to the ${ }^{125} \mathrm{I}-\mathrm{SHPP}$, the $\mathrm{pH}$ was adjusted to 9 with $1 \mathrm{M} \mathrm{Na}_{2} \mathrm{CO}_{3}$, and the reaction mixture was incubated at room temperature for 30-60 min. After incubation, the labeling yield was determined by radio-instant thin-layer chromatography. The ${ }^{125}$ I-labeled $\mathrm{mAb}$ was purified twice (Zeba spin desalting columns [Pierce Protein
Research], 40-kDa molecular weight cutoff), and the radiochemical purity of the ${ }^{125}$ I-CC49-TCO solution was determined by radio-instant thin-layer chromatography, size-exclusion high-performance liquid chromatography, and sodium dodecyl sulfate polyacrylamide gel electrophoresis. For animal experiments, the specific activity of the ${ }^{125} \mathrm{I}-$ $\mathrm{CC} 49-\mathrm{TCO}$ was adjusted to $2-4 \mathrm{kBq} / \mu \mathrm{g}$ by adding nonradioactive CC49-TCO.

\section{Animal Experiments}

All experiments were performed on tumor-free or tumor-bearing nude female BALB/C mice (20- to 25-g body weight; Charles River Laboratories) according to the principles of laboratory animal care (13) and the Dutch national law "Wet op de Dierproeven" (Staatsblad 1985, 336). Mice were inoculated subcutaneously with $5 \times 10^{6}$ LS174T cells in $100 \mu \mathrm{L}$ of sterile phosphate-buffered saline and were used 7-10 d later, when the tumors reached $70-200 \mathrm{~mm}^{3}$. The mice were euthanized by cervical dislocation, blood was withdrawn, and selected organs and tissues were harvested and blotted dry. All samples were weighed and then combined with $1 \mathrm{~mL}$ of phosphate-buffered saline. The sample radioactivity was counted in a $\gamma$ counter (Wizard 1480; PerkinElmer) along with standards to determine the percentage injected dose (\%ID) per gram and the \%ID per organ. The tissues from dual-isotope experiments were measured using energy windows of 10-80 $\mathrm{keV}$ and $155-380 \mathrm{keV}$ for ${ }^{125} \mathrm{I}$ and ${ }^{177} \mathrm{Lu}$, respectively, with cross-contamination correction.

\section{CC49-TCO Blood Clearance with CA Administration}

Two groups of 3 tumor-free mice were injected with ${ }^{125}$ I-labeled CC49-TCO (average of $9 \mathrm{TCOs} / \mathrm{mAb}, 20 \mu \mathrm{g} / 100 \mu \mathrm{L}$ per mouse; $\sim 0.2 \mathrm{MBq})$, and blood samples $(\sim 20 \mu \mathrm{L})$ were withdrawn from the vena saphena after 5 and $30 \mathrm{~min}$. Thirty-five minutes after mAb injection, each mouse received an intravenous injection of $3(120 \mu \mathrm{g} / 100 \mu \mathrm{L}$ per mouse $)$ or $4\left(4 \times 10^{7}\right.$ beads $/ 100 \mu \mathrm{L}$ per mouse $)$. More blood samples were withdrawn, and the animals were euthanized 210 min after mAb injection. One more group of 3 tumor-free mice was injected intravenously with ${ }^{125} \mathrm{I}$-labeled CC49-TCO $(100 \mu \mathrm{g} / 100 \mu \mathrm{L}$ per mouse,

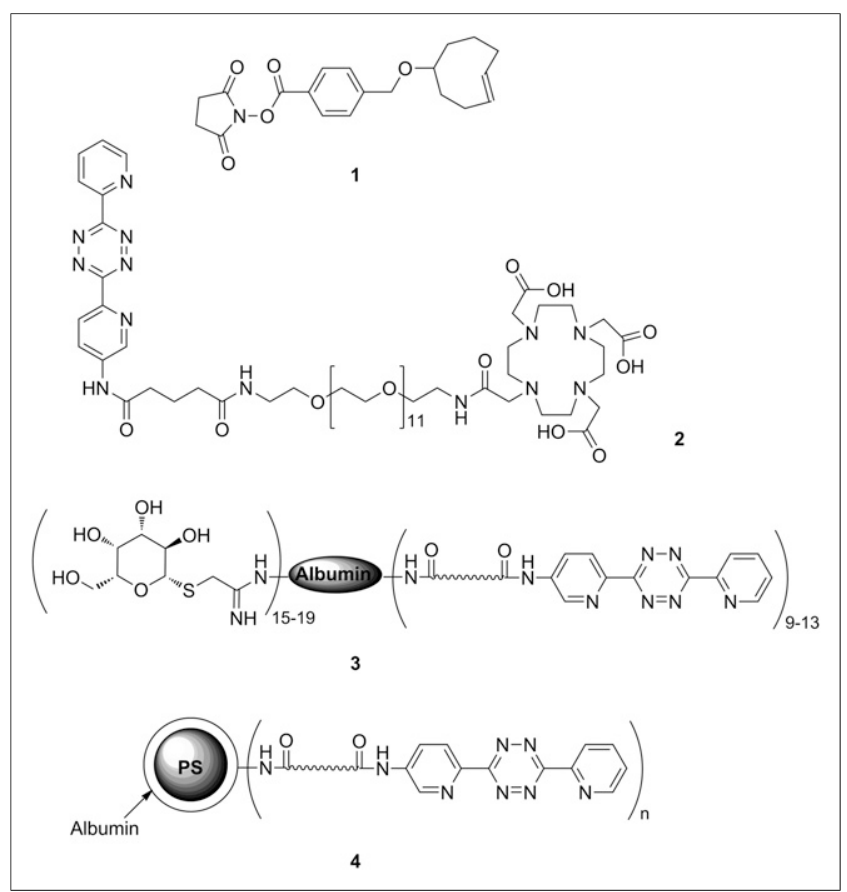

FIGURE 2. Pretargeting components:TCO-NHS(1) and DOTA-tetrazine (2) and schematic representations of galactose-albumin-tetrazine (3) and polystyrene (PS) beads coated with tetrazine-conjugated albumin (4). 
$\sim 0.4 \mathrm{MBq}$ ). The mice were serially bled at selected times and euthanized $4 \mathrm{~d}$ after $\mathrm{mAb}$ injection.

\section{Pretargeting Protocol and SPECT Imaging}

Two groups of 4 tumor-bearing mice were injected with ${ }^{125}$ I-labeled CC49-TCO (average of $7 \mathrm{TCOs} / \mathrm{mAb}, 100 \mu \mathrm{g} / 100 \mu \mathrm{L}$ per mouse; $\sim 0.4 \mathrm{MBq}$ ) followed by 1 dose of $330 \mathrm{~h}$ after mAb injection or by 2 doses 30 and $48 \mathrm{~h}$ after $\mathrm{mAb}$ injection. Two hours after the (last) CA injection, the mice received $6.7 \mathrm{nmol}$ of ${ }^{177} \mathrm{Lu}-2(8.52 \mu \mathrm{g} / 80 \mu \mathrm{L}$ containing $100 \mu \mathrm{g}$ of gentisic acid; $\sim 0.5 \mathrm{MBq}$ ) and were euthanized $3 \mathrm{~h}$ after tetrazine injection, after which organs and tissues of interest were counted for radioactivity.

For SPECT/CT imaging, a tumor-bearing mouse was injected with CC49-TCO, compound 3, and ${ }^{111} \mathrm{In}-2(6.7 \mathrm{nmol} / 80 \mu \mathrm{L} ; \sim 42 \mathrm{MBq})$ following the optimized protocol. Approximately $90 \mathrm{~min}$ after tetrazine injection, the mouse was anesthetized with isoflurane and imaged on a small-animal SPECT/CT system (NanoSPECT/CT; Bioscan). Three hours after tetrazine injection, the mouse was euthanized by anesthesia overdose and a high-resolution SPECT/CT scan was obtained. The postmortem SPECT data were quantified by drawing volumes of interest in tumor, kidneys, liver, and thigh muscle using InVivoScope (Bioscan).

\section{Dosimetry}

Groups of 4 tumor-bearing mice were injected either with ${ }^{177} \mathrm{Lu}-$ DOTA-CC49 $(100 \mu \mathrm{g} / 100 \mu \mathrm{L}$ per mouse; $\sim 0.5 \mathrm{MBq}$, supplemental data) or according to the optimized pretargeting protocol. One group of mice injected with ${ }^{177} \mathrm{Lu}$-DOTA-CC49 and one injected with pretargeted ${ }^{177} \mathrm{Lu}$-tetrazine 2 were used to evaluate the early blood distribution of the 2 tracers and were then euthanized 3 and $1 \mathrm{~h}$ after ${ }^{177} \mathrm{Lu}-\mathrm{DOTA}-\mathrm{CC} 49$ and ${ }^{177} \mathrm{Lu}-2$, respectively. All other groups were euthanized at various times up to $7 \mathrm{~d}$ after tracer injection. Two additional groups of tumor-bearing mice were used for excretion profiles. Radiation dosimetry was estimated for a male human model (standard 73-kg adult) and for a voxelized mouse model using the biodistribution data according to the MIRD methodology. Activity concentration $(\% \mathrm{ID} / \mathrm{g})$ in selected tissues was converted to \% ID per organ and corrected for radioactive decay to the time of injection. The time-activity curves were fitted by a 2-phase exponential clearance function or by the trapeze method and integrated analytically to provide the organ residence times. The time-activity curve of the tumor was fitted by a monoexponential uptake function. The residence time of the red marrow was calculated as a fraction of the blood residence time $\left(\tau_{\text {marrow }}=0.08 \tau_{\text {blood }}\right)$.

Radiation doses to the mouse organs were evaluated using the MIRD methodology, where the $S$ factors $\left(S_{\text {target } \leftarrow \text { source }}\right)$ were calculated using a voxelized model of a mouse and the electron transport program EGSnrc (National Research Council Canada) to calculate energy deposition. The 4-dimensional digital mouse whole-body phantom of Segars et al. (14) was voxelized on a $0.2-\mathrm{mm}$ grid and modified to include intestine wall and content, stomach wall and content, bladder wall and content, a $20-\mu \mathrm{L}$ gallbladder, and a 0.53 -g tumor (left side of the neck). Twenty-seven independent simulations, each for $10^{7}$ decays, were performed with the program EGSnrc to calculate the energy deposition in each target organ for each source organ. The simulation included all decay particles from ${ }^{177} \mathrm{Lu}$ (www.nndc.bnl. gov/MIRD). Human radiation dose estimates were calculated using the residence times of ${ }^{177} \mathrm{Lu}-\mathrm{DOTA}-\mathrm{CC} 49$ and ${ }^{177} \mathrm{Lu}-2$ and the OLINDA/EXM program.

\section{Data Analysis}

The data are presented as mean \%ID per gram or per organ \pm 1 SD. Curve fitting and 2-tailed unpaired $t$ tests were performed with Prism, version 4.1 (GraphPad). The difference between 2 data was considered statistically significant when the $P$ value was less than 0.05 . The error bars on the mouse and human radiation dose estimates are commensurate with the errors on the residence times (Supplemental Table 3) and range from $20 \%$ to $50 \%$.

\section{RESULTS}

\section{Component Development}

The TAG-72-targeting mAb CC49 was reacted with a new TCO- $N$-hydroxysuccinimide (NHS) derivative with increased reactivity and in vivo stability $\left(\mathbf{1}, k_{2}=2.7 \pm 0.0 \times 10^{5} \mathrm{M}^{-1} \mathrm{~s}^{-1}\right.$; 3.94-d stability half-life in vivo (12)), which afforded an average of 7-9 TCO groups per mAb. All radiolabeled mAb constructs were of greater than $98 \%$ purity.

Both CAs were evaluated in vivo with respect to their mAbTCO binding and clearing efficacy. In tumor-free mice, ${ }^{125} \mathrm{I}-$ CC49-TCO $(100 \mu \mathrm{g})$ without CA exhibited an expected slow blood clearance $\left(\mathrm{t}_{1 / 2, \alpha}=2.0 \mathrm{~h} ; \mathrm{t}_{1 / 2, \beta}=23.5 \mathrm{~h}\right.$; Fig. 3C). To test whether the CAs would effectively remove a residual amount of circulating $\mathrm{mAb}$ several days after injection, a lower amount (20 $\mu \mathrm{g})$ of ${ }^{125} \mathrm{I}-\mathrm{CC} 49-\mathrm{TCO}$ was injected followed by $\mathbf{3}$ or $\mathbf{4}$ after $35 \mathrm{~min}$. Within $30 \mathrm{~min}$, the ${ }^{125} \mathrm{I}-\mathrm{CC} 49-\mathrm{TCO}$ blood levels diminished, respectively, 22-fold (to $1.62 \pm 0.35 \% \mathrm{ID} / \mathrm{g}$, Fig. 3A) and 12-fold (to $2.70 \pm 0.40 \% \mathrm{ID} / \mathrm{g}$, Fig. 3B). However, for 4 a small increase in blood radioactivity to approximately $4 \% \mathrm{ID} / \mathrm{g}$ was observed after the initial drop. No such rebound effect was detected in mice treated with $\mathbf{3}$. The subsequent biodistribution study (Fig. 3D) showed most of the radioactivity in the liver $(12.05 \pm 1.29 \% \mathrm{ID} / \mathrm{g})$ and minor uptake in the spleen $(2.37 \pm$ $0.53 \% \mathrm{ID} / \mathrm{g}$ ) of the mice treated with 3 , and for 4 high uptake in both liver and spleen $(18.55 \pm 3.51$ and $9.55 \pm 2.77 \% \mathrm{ID} / \mathrm{g}$, respectively). On the basis of these results, CA 3 was chosen for further evaluation.

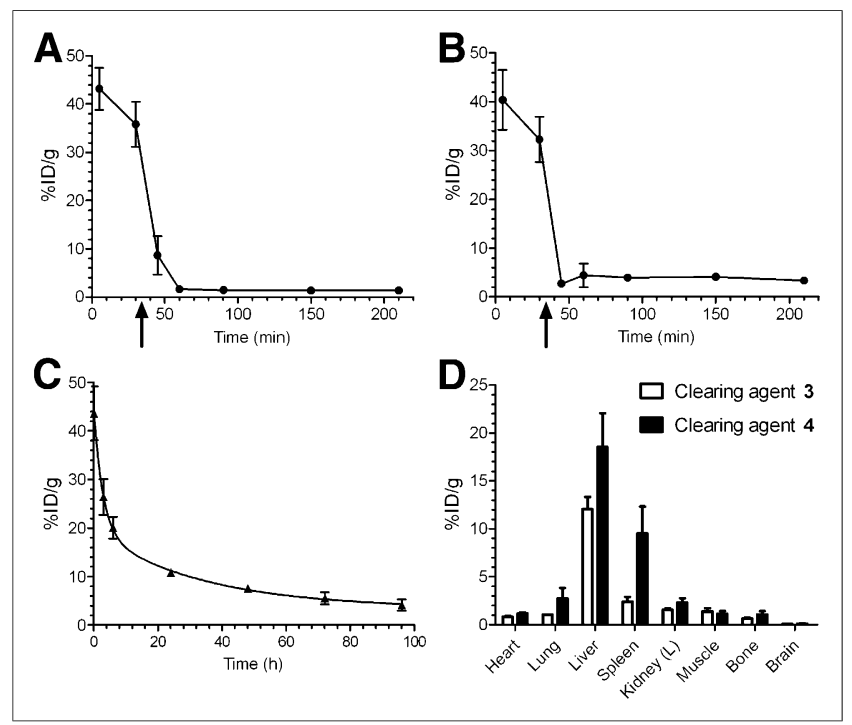

FIGURE 3. Blood clearance of $125 \mathrm{I}-\mathrm{CC} 49-\mathrm{TCO}$ with and without use of CAs: $20 \mu \mathrm{g}$ of mAb per mouse followed by $120 \mu \mathrm{g}$ of galactose-albumintetrazine (3) (A), $20 \mu \mathrm{g}$ of $\mathrm{mAb}$ followed by $4 \times 10^{7}$ beads coated with albumin-tetrazine (4) (arrows indicate time of CA injection) (B), $100 \mu \mathrm{g}$ of $\mathrm{mAb}$ per mouse without CA (C), and biodistribution of ${ }^{125}$-CC 49 -TCO 210 min after mAb injection and after $\mathbf{3}$ or $\mathbf{4}$ (D). Data points represent mean $\% \mathrm{ID} / \mathrm{g} \pm \mathrm{SD}(n=3)$. 


\section{Pretargeting Protocol Optimization and SPECT/CT Imaging}

From a dosing study, we found $160 \mu \mathrm{g}$ of $\mathbf{3}$ per mouse to give the optimal clearing efficacy (supplemental data). To further increase the T/B ratio, we then evaluated the effect of a single dose versus a double dose of $\mathbf{3}$ on the blood and tumor content of both pretargeting components, ${ }^{125} \mathrm{I}-\mathrm{CC} 49-\mathrm{TCO}$ and ${ }^{177} \mathrm{Lu}-\mathbf{2}$, in a dualisotope biodistribution experiment (Fig. 4). Tumor-bearing mice received intravenous injections of 1 or $2 \mathrm{CA}$ doses $(30 \mathrm{~h}$, or 30 and $48 \mathrm{~h}$ after mAb injection) followed by ${ }^{177} \mathrm{Lu}-2 \mathrm{~h}$ after the last dose and were euthanized $3 \mathrm{~h}$ later. A single CA dose already reduced the ${ }^{125} \mathrm{I}-\mathrm{CC} 49-\mathrm{TCO}$ in blood to $1.16 \pm 0.43 \% \mathrm{ID} / \mathrm{g}$ (Fig. 4A), compared with the $8.47 \pm 4.12 \% \mathrm{ID} / \mathrm{g}$ we previously had to contend with without CA. Also, only low levels of ${ }^{125} \mathrm{I}$ radioactivity were detected in blood-rich tissues such as heart and lung. The radioactivity in the liver $5 \mathrm{~h}$ after CA injection $(4.20 \pm 1.16 \% \mathrm{ID} / \mathrm{g})$ was significantly lower than that found at $3 \mathrm{~h}$ in the initial biodistribution experiment $(12.05 \pm 1.29$ $\%$ ID/g; Fig. 3D), supporting the rapid 3/ ${ }^{125} \mathrm{I}-\mathrm{CC} 49-\mathrm{TCO}$ metabolism and radioiodine elimination from the hepatocytes (15). To our satisfaction, a second dose of compound $\mathbf{3}$ further reduced the amount of ${ }^{125} \mathrm{I}-\mathrm{CC} 49-\mathrm{TCO}$ in blood $(0.19 \pm 0.04 \% \mathrm{ID} / \mathrm{g})$ and in all other considered organs, and the T/B, tumor-to-liver, and tumor-to-spleen ratios doubled or tripled (Supplemental Fig. 4A).

The elimination of non-tumor-bound CC49-TCO translated into a greatly improved distribution of ${ }^{177} \mathrm{Lu}-2$ (Fig. 4B). We reduced the amount of tetrazine from the $17 \mathrm{nmol}$ used in our previous studies without CA (8) to $6.7 \mathrm{nmol}$ (1.2 molar equivalents with respect to TCO). As a result of this factor and the reduced CC49TCO in circulation, tumor uptake increased from $3.12 \pm 0.87 \% \mathrm{ID} / \mathrm{g}$ to $7.45 \pm 1.46 \% \mathrm{ID} / \mathrm{g}$ and $6.13 \pm 1.09 \% \mathrm{ID} / \mathrm{g}$ with 1 and $2 \mathrm{CA}$ doses, respectively. Importantly, the administration of a second CA dose significantly increased the tumor-to-nontumor ratios for blood (254 vs. 46 ), muscle (246 vs. 128), and other tissues (Supplemental Fig. 4B).

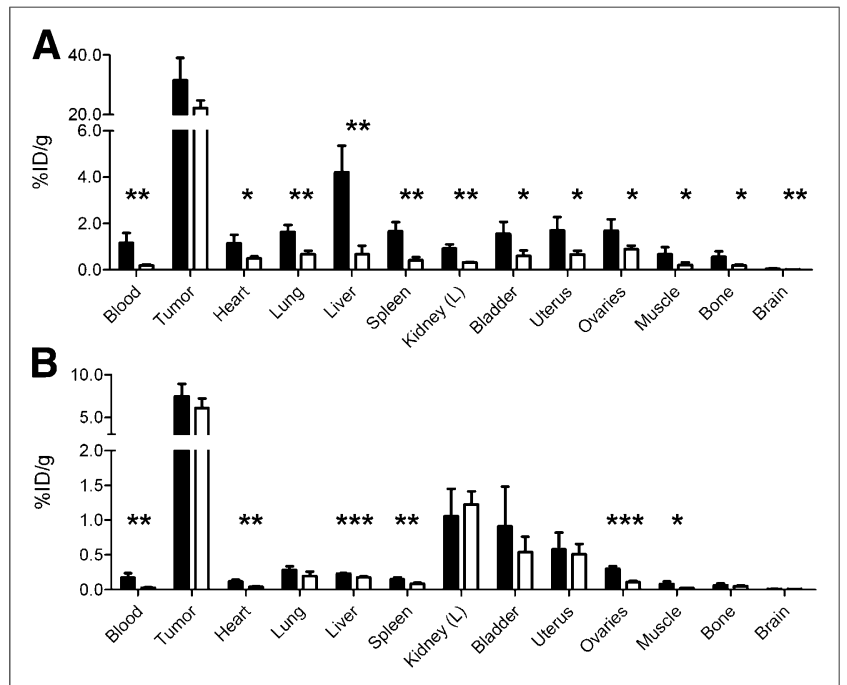

FIGURE 4. Dual-isotope biodistribution of 125 -CC49-TCO (A) and ${ }^{177}$ Lu-tetrazine 2 (B) in LS174T tumor-bearing mice treated with 1 dose ( $30 \mathrm{~h}$ after mAb injection, solid bars) or 2 doses (30 and $48 \mathrm{~h}$ after mAb injection, open bars) of galactose-albumin-tetrazine (3). Mice were injected with ${ }^{177} \mathrm{Lu}-22 \mathrm{~h}$ after last CA and were euthanized $3 \mathrm{~h}$ later. Bars represent mean \% ID/g \pm SD $(n=4)$. ${ }^{*} P<0.05$. ${ }^{* \star} P<0.005$. ${ }^{\star * *} P<0.001$.

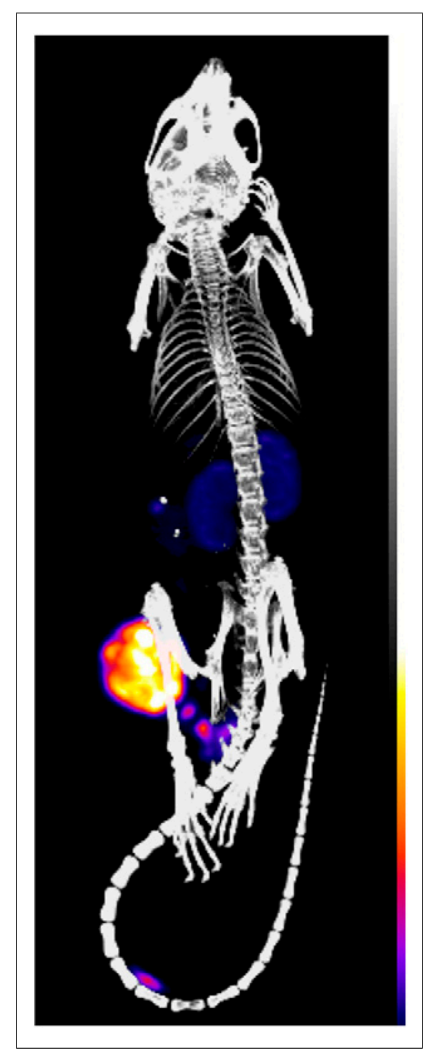

FIGURE 5. SPECT/CT image (postmortem, maximum intensity projection) of CC49-TCO pretargeted mouse bearing LS174T xenograft, $3 \mathrm{~h}$ after ${ }^{111} \mathrm{In}$-tetrazine 2 injection. SPECT/CT image of live mouse is available as Supplemental Figure 5. biliary mAb elimination, but only $18.66 \pm 2.26 \%$ ID had been excreted $4 \mathrm{~d}$ after ${ }^{177} \mathrm{Lu}-\mathrm{DOTA}-\mathrm{CC} 49$ injection.

In contrast, in mice pretreated with $\mathrm{CC} 49-\mathrm{TCO},{ }^{177} \mathrm{Lu}-2$ cleared rapidly from the blood, with an 11-min half-life (Supplemental Fig. 6B) and all nonreacted probe was eliminated via the urine $(93.75 \pm 3.15 \%$ ID $1 \mathrm{~h}$ after injection). After only $1 \mathrm{~h}$, the tumor exhibited the highest ${ }^{177} \mathrm{Lu}-2$ uptake $(4.71 \pm 1.13 \% \mathrm{ID} / \mathrm{g})$ among the considered tissues, and tumor uptake peaked $(5.38 \pm 0.48$ $\% \mathrm{ID} / \mathrm{g}$ ) at $3 \mathrm{~h}$ whereas the remaining activity in blood, muscle, and bone decreased rapidly (Table 2 and Supplemental Table 2).

These mouse biodistribution and excretion data were used to estimate the absorbed doses to tumor and normal organs in mice and to normal organs in humans. For both procedures, the doselimiting organ in mice is the bone marrow. As expected, the estimated dose to the tumor in mice (Table 3) injected with directly labeled CC49 is high $(9,573 \mathrm{mGy} / \mathrm{MBq})$, but the bone marrow and the liver also receive high doses: respectively, 1,136 and $942 \mathrm{mGy} / \mathrm{MBq}$. Although the estimated dose to the tumor in mice injected with pretargeted ${ }^{177} \mathrm{Lu}$-tetrazine is lower $(479 \mathrm{mGy} /$ $\mathrm{MBq}$ ), much lower doses are delivered to the bone marrow (7 $\mathrm{mGy} / \mathrm{MBq}$ ) and to all other nontarget organs and tissues, including those of the urinary tract. In a standard adult man treated with pretargeted ${ }^{177} \mathrm{Lu}$-tetrazine, the estimated dose in most organs is 1-2 orders of magnitude lower than with ${ }^{177} \mathrm{Lu}$-DOTA-CC49 (Table 4). The primary and secondary critical organs for 
TABLE 1

Tumor-to-Organ Ratios for ${ }^{177}$ Lu-DOTA-CC49 in Mice Bearing LS174T Colon Carcinoma Xenografts

\begin{tabular}{|c|c|c|c|c|c|c|c|}
\hline Organ & $3 \mathrm{~h}$ & $6 \mathrm{~h}$ & $1 d$ & $2 d$ & $3 d$ & $4 d$ & $7 \mathrm{~d}^{*}$ \\
\hline Blood & $<1$ & $<1$ & $3 \pm 0$ & $5 \pm 1$ & $13 \pm 3$ & $31 \pm 11$ & $21 \pm 3$ \\
\hline Liver & $2 \pm 0$ & $2 \pm 0$ & $6 \pm 2$ & $7 \pm 3$ & $14 \pm 6$ & $3 \pm 1$ & $8 \pm 2$ \\
\hline Kidney & $2 \pm 0$ & $3 \pm 0$ & $12 \pm 2$ & $14 \pm 2$ & $26 \pm 3$ & $38 \pm 3$ & $44 \pm 4$ \\
\hline Bladder & $14 \pm 3$ & $13 \pm 6$ & $14 \pm 3$ & $16 \pm 4$ & $35 \pm 7$ & $63 \pm 14$ & $61 \pm 4$ \\
\hline Muscle & $9 \pm 2$ & $21 \pm 7$ & $36 \pm 13$ & $44 \pm 14$ & $101 \pm 12$ & $219 \pm 71$ & $187 \pm 10$ \\
\hline Bone & $6 \pm 1$ & $11 \pm 1$ & $27 \pm 4$ & $39 \pm 2$ & $53 \pm 7$ & $85 \pm 16$ & $101 \pm 6$ \\
\hline $\begin{array}{l}{ }^{*} n=3 . \\
\text { Data are }\end{array}$ & $\mathrm{SD}(n=$ & & & & & & \\
\hline
\end{tabular}

${ }^{177} \mathrm{Lu}-\mathrm{DOTA}-\mathrm{CC} 49$ are the liver $(1.33 \mathrm{mGy} / \mathrm{MBq})$ and ovaries $(1.13 \mathrm{mGy} / \mathrm{mBq})$, whereas those for pretargeted ${ }^{177} \mathrm{Lu}$-tetrazine are the bladder wall $(0.104 \mathrm{mGy} / \mathrm{MBq})$ and the kidneys $(0.0425$ $\mathrm{mGy} / \mathrm{MBq})$. The effective dose for intact $\mathrm{mAb}$ and pretargeted tetrazine is 0.447 and $0.0195 \mathrm{mSv} / \mathrm{MBq}$, respectively.

\section{DISCUSSION}

We sought to develop a CA capable of eliminating circulating $\mathrm{mAb}$-TCO to explore the full therapeutic potential of tumor pretargeting with the inverse-electron-demand Diels-Alder reaction. In pretargeting, CAs should rapidly bind the mAb in blood and remove it from circulation to the liver or spleen without blocking the tumor-bound mAb. Therefore, they should not diffuse out of the vascular compartment or produce tag-binding species on catabolism. We developed 2 types of agent that function through active or passive liver targeting (Fig. 2). For active liver targeting, we turned to galactose-mediated liver uptake via Ashwell receptors on hepatocytes, which has successfully been used in the clinic for biotin- and galactose-functionalized albumin and dendrimer CAs (11). We synthesized an albumin scaffold carrying tetrazine and galactose moieties (compound $\mathbf{3}$ ) for reaction with the mAb-bound TCO and interaction with the Ashwell receptors in liver, respectively. For passive liver targeting, we used polymer microparticles, because most intravenously injected particles clear via the reticuloendothelial system within minutes (16). In addition, the particle size precludes extravasation from the central vascular compartment and allows for high tetrazine loading. We prepared an albumintetrazine conjugate and used it to coat the surface of polystyrene beads (compound 4 ) to be used as a model particulate CA.

In a preliminary evaluation in tumor-free mice, both CAs were capable of removing ${ }^{125} \mathrm{I}-\mathrm{CC} 49-\mathrm{TCO}$ from the circulation (Figs.
$3 \mathrm{~A}$ and $3 \mathrm{~B}$ ), thus indicating that an efficient reaction occurred between the tetrazine groups on the CAs and the TCO on the $\mathrm{mAb}$ in blood. However, for $\mathbf{4}$ a small increase in blood radioactivity was observed after the initial drop, possibly caused by CC49-TCO reequilibration from physiologic compartments (17). This rebound was not due to ${ }^{125} \mathrm{I}$ recirculation: at the time of mouse euthanasia the activity in stomachs and thyroids was low $(1.66 \pm 0.70$ and $0.59 \pm 0.15 \% \mathrm{ID} /$ organ $)$, confirming in vivo stability of the iodinated mAb. No such rebound effect was detected in mice treated with $\mathbf{3}$, possibly because, on reequilibration, galactosylated albumin is still present in the circulation to some extent whereas the even more quickly clearing microparticles are not $(18,19)$. The subsequent biodistribution study (Fig. 3D) confirmed the clearing mechanisms of both agents, with most of the radioactivity in the mice treated with $\mathbf{3}$ found in the liver whereas for $\mathbf{4}$ a high uptake was found in both liver and spleen, the main organs of the reticuloendothelial system. From the radioactivity in the intestine $(22.83 \pm 4.92 \%$ ID/organ with 3 vs. $12.97 \pm$ $1.82 \%$ ID/organ with 4 ), it appears that mice treated with 3 excreted radioactivity more quickly than those that received 4 . On the basis of this preliminary evaluation, we chose to include $\mathbf{3}$ in an optimized pretargeting protocol.

After determining the optimal dose of CA 3, we continued with a comparison between a single and double CA dose in a dual-isotope biodistribution experiment (Fig. 4). The increased stability of the new TCO (12) allowed for a longer interval between mAb and probe administration than was possible with our previous experiments. We also reduced the amount of injected tetrazine 2 (from 17 to $6.7 \mathrm{nmol} / \mathrm{mouse}$, that is, 1.2 equivalents with respect to $\mathrm{TCO})$ to increase the radioactivity $\% \mathrm{ID} / \mathrm{g}$ in tumor. As a result of the reduction in TCO blood concentration from about $0.4 \mu \mathrm{M}$ when not using a CA to 50 and $10 \mathrm{nM}$ with 1 and 2 doses of CA $\mathbf{3}$,

TABLE 2

Tumor-to-Organ Ratios for CC49-TCO-Pretargeted ${ }^{177}$ Lu-Tetrazine 2 in Mice Bearing LS174T Colon Carcinoma Xenografts

\begin{tabular}{|c|c|c|c|c|c|c|c|}
\hline Organ & $1 \mathrm{~h}$ & $3 \mathrm{~h}$ & $6 \mathrm{~h}$ & $1 \mathrm{~d}$ & $2 d$ & $4 d$ & $7 d$ \\
\hline Blood & $13 \pm 6$ & $196 \pm 13$ & $206 \pm 75$ & $307 \pm 90$ & $410 \pm 270$ & $446 \pm 82$ & $540 \pm 169^{*}$ \\
\hline Liver & $17 \pm 6$ & $31 \pm 3$ & $30 \pm 9$ & $30 \pm 8$ & $30 \pm 18$ & $14 \pm 4$ & $8 \pm 1$ \\
\hline Kidney & $2 \pm 1$ & $3 \pm 0$ & $4 \pm 1$ & $4 \pm 1$ & $7 \pm 3$ & $6 \pm 2$ & $6 \pm 2$ \\
\hline Bladder & $3 \pm 2$ & $8 \pm 4$ & $25 \pm 10$ & $20 \pm 7$ & $33 \pm 16$ & $15 \pm 3$ & $7 \pm 2$ \\
\hline Muscle & $31 \pm 18$ & $141 \pm 67$ & $245 \pm 108$ & $257 \pm 75$ & $229 \pm 145$ & $203 \pm 100$ & $255 \pm 152$ \\
\hline Bone & $23 \pm 20^{*}$ & $95 \pm 86$ & $162 \pm 46$ & $159 \pm 69$ & $149 \pm 78$ & $114 \pm 61$ & $76 \pm 25$ \\
\hline $\begin{array}{l}{ }^{*} n=3 . \\
\text { Data are }\end{array}$ & $\pm \mathrm{SD}(n=$ & & & & & & \\
\hline
\end{tabular}


TABLE 3

Radiation Dose in Mouse Organs and Tissues in mGy/MBq

\begin{tabular}{|lrc}
\hline \multicolumn{1}{c}{ Organ } & ${ }^{177}$ Lu-DOTA-CC49 & 177 Lu-tetrazine \\
\hline Lungs & 189 & 5 \\
\hline Heart wall & 347 & 2 \\
\hline Kidneys & 328 & 58 \\
\hline Liver & 942 & 10 \\
\hline Pancreas & 57 & 2 \\
\hline Spleen & 460 & 15 \\
\hline Intestine wall & 124 & 9 \\
\hline Brain & 45 & 1 \\
\hline Stomach wall & 302 & 16 \\
\hline Testes & 78 & 1 \\
\hline Ribs & 333 & 8 \\
\hline Thyroid & 114 & 1 \\
\hline Femur & 261 & 8 \\
\hline Cranium & 404 & 13 \\
\hline Trachea & 153 & 2 \\
\hline Spine & 475 & 8 \\
\hline Spine (marrow) & 1136 & 7 \\
\hline Bladder wall & 354 & 168 \\
\hline Skin & 62 & 1 \\
\hline Gallbladder & 157 & 2 \\
\hline Tumor & 9573 & 479 \\
\hline Whole body & 601 & 5 \\
\hline Effective dose (mSv/MBq) & 344 & 15.6 \\
\hline
\end{tabular}

respectively, the ${ }^{177} \mathrm{Lu}-2$ retention in blood at $3 \mathrm{~h}$ after injection was extremely low (respectively, $0.17 \pm 0.06$ and $0.03 \pm 0.01$ $\% \mathrm{ID} / \mathrm{g}$ ) and accordingly the $\mathrm{T} / \mathrm{B}$ ratio increased exponentially, from about 2 without a CA to 80 with $1 \mathrm{CA}$ dose to 250 with 2 doses (Supplemental Fig. 4). This 125-fold improvement in T/B ratio, and the biodistribution profile in general are within the range of values achieved in mice with the established, biologic, pretargeting methods (20-23).

These encouraging results prompted us to conduct a dosimetry comparison between direct radioimmunotherapy with ${ }^{177} \mathrm{Lu}-$ DOTA-CC49 and our optimized chemical pretargeting approach. The typical mAb biodistribution data obtained for ${ }^{177} \mathrm{Lu}-\mathrm{DOTA}-$ CC49, with a sustained circulation, high tumor uptake, and substantial radioactivity retention in blood-rich organs and in excretory organs, were in good agreement with those previously published for other ${ }^{177} \mathrm{Lu}-\mathrm{CC} 49$ constructs (24). In contrast, in mice pretreated with CC49-TCO, ${ }^{177} \mathrm{Lu}-2$ cleared rapidly from blood and all nonreacted probe was eliminated via the urine within a few hours. The apparent decrease in tumor values after the peak ${ }^{177} \mathrm{Lu}-$ 2 uptake in tumor $3 \mathrm{~h}$ after injection $(5.38 \pm 0.48 \% \mathrm{ID} / \mathrm{g})$ is a consequence of radioactivity dilution due to tumor growth from approximately 0.4 to $1.5 \mathrm{~g}$ in $7 \mathrm{~d}$. The absolute amount of ${ }^{177} \mathrm{Lu}$ in the whole tumor $\left(2.16 \pm 0.43 \%\right.$ ID $3 \mathrm{~h}$ after ${ }^{177} \mathrm{Lu}-2$ injection $) \mathrm{did}$ not decrease significantly with time, because $1.49 \pm 0.56 \%$ ID was still present in the tumor $7 \mathrm{~d}$ later. This is a significant difference when compared with results obtained with CC49-scFvstreptavidin fusion protein followed by ${ }^{177} \mathrm{Lu}$-DOTA-biotin (25, 26). For instance, Lewis et al. (26) observed a 10-fold decrease in ${ }^{177} \mathrm{Lu} \% \mathrm{ID} / \mathrm{g}$ in LS174T xenografts over $1 \mathrm{wk}$, unrelated to tumor growth. Our findings suggest strong binding of the CC49-TCO to TAG-72 in the tumor and in vivo stability of the inverse-electrondemand Diels-Alder reaction product between the tumor-bound TCO and the tetrazine, which are essential features for the delivery of a therapeutic dose.
Besides the tumor, the kidney was the only organ to retain the ${ }^{177} \mathrm{Lu}-2$, albeit to a lesser extent $(1.58 \pm 0.14 \% \mathrm{ID} / \mathrm{g}$ at $3 \mathrm{~h})$ and less than previously found for ${ }^{177} \mathrm{Lu}$ - and ${ }^{90}$ Y-labeled biotin (20, 26). As a result of the good tumor uptake and fast clearance from blood in the pretargeted mice, the T/B ratio was approximately 200 already at $3 \mathrm{~h}$ after ${ }^{177} \mathrm{Lu}-2$ injection and increased to more than 500 by the end of the experiment (Table 2). In contrast, the T/B ratio for ${ }^{177} \mathrm{Lu}-\mathrm{DOTA}-\mathrm{CC} 49$ exceeded 1 only $1 \mathrm{~d}$ after $\mathrm{mAb}$ injection and $3 \mathrm{~d}$ later peaked at about 30 (Table 1). The rapid and selective reaction of the tetrazine with the chemically tagged tumors, and the resulting excellent tumor-to-nontumor ratios, were also demonstrated in a corresponding SPECT/CT imaging study of live mice using ${ }^{111}$ In-2 (Fig. 5 and Supplemental Fig. 5).

The estimated radiation doses to the mouse organs (Table 3) were derived from the biodistribution data using the MIRD methodology. The dose-limiting organ for both ${ }^{177} \mathrm{Lu}-\mathrm{CC} 49$ and pretargeted ${ }^{177} \mathrm{Lu}$-tetrazine was the bone marrow. On the basis of Table 3 and the maximum tolerated dose of 2.5 Gy for marrow (27), we estimated that less than 21 Gy would be delivered to the tumor with ${ }^{177} \mathrm{Lu}-\mathrm{CC} 49$ whereas the pretargeting approach would deliver 8-fold more, 171 Gy, exceeding 80-100 Gy, commonly considered the threshold for clinical efficacy for radioimmunotherapy of solid tumors (28). Human dosimetry indicated that, similar to mice, humans treated with pretargeted ${ }^{177} \mathrm{Lu}-2$ would receive a dose to nontarget tissues 1 to 2 orders of magnitude lower than with ${ }^{177}$ Lu-CC49 (Table 4). Our results suggest that chemical pretargeting can deliver to the tumor a therapeutic radiation dose up to 1 order of magnitude higher than nonpretarged radioimmunotherapy, which is on a par with the results from biologic pretargeting studies in mice $(20,21,23,29)$.

TABLE 4

Organ Radiation Doses in Adult Men $(73 \mathrm{~kg})$ in mGy/MBq

\begin{tabular}{lll}
\hline \multicolumn{1}{c}{ Organ } & 177Lu-DOTA-CC49 & 177Lu-tetrazine \\
\hline Adrenals & 0.172 & 0.00162 \\
\hline Brain & 0.0177 & 0.000146 \\
\hline Breasts & 0.154 & 0.0013 \\
\hline Gallbladder wall & 0.184 & 0.0018 \\
\hline Lower large intestine & 0.354 & 0.0363 \\
\hline Small intestine & 0.319 & 0.0101 \\
\hline Stomach wall & 0.208 & 0.00599 \\
\hline Upper large intestine & 0.286 & 0.0231 \\
\hline Heart wall & 0.0944 & 0.000537 \\
\hline Kidneys & 0.427 & 0.0425 \\
\hline Liver & 1.33 & 0.0142 \\
\hline Lungs & 0.0894 & 0.00288 \\
\hline Muscle & 0.0466 & 0.000861 \\
\hline Ovaries & 1.13 & 0.0341 \\
\hline Pancreas & 0.172 & 0.00164 \\
\hline Red marrow & 0.213 & 0.00302 \\
\hline Osteogenic cells & 0.799 & 0.00172 \\
\hline Skin & 0.150 & 0.0029 \\
\hline Spleen & 0.348 & 0.0112 \\
\hline Testes & 0.155 & 0.00151 \\
\hline Thymus & 0.158 & 0.00134 \\
\hline Thyroid & 0.157 & 0.00132 \\
\hline Bladder wall & 0.261 & 0.104 \\
\hline Uterus & 0.600 & 0.0293 \\
\hline Effective dose (mSv/MBq) & 0.217 & 0.00287 \\
\hline & 0.447 & 0.0195 \\
\hline
\end{tabular}




\section{CONCLUSION}

The use of a rapid bioorthogonal clearing approach and an optimized chemical pretargeting protocol, combined with a TCO with increased stability and a high reactivity of $k_{2}=2.7 \pm 0.0 \times 10^{5}$ $\mathrm{M}^{-1} \mathrm{~S}^{-1}$, resulted in a selective and effective on-tumor reaction with, and retention of, a small and quickly clearing radiolabeled probe and high tumor-to-nontumor ratios. The predicted 8 -fold higher total tumor dose of this approach compared with conventional radioimmunotherapy is similar to results obtained in mice with the clinically validated, noncovalent pretargeting approaches, which have association rates ranging from $5 \times 10^{5}$ to $7.5 \times 10^{7}$ $\mathrm{M}^{-1} \mathrm{~S}^{-1}$. The chemical components are less likely to give rise to immunogenicity, potentially enabling repeated treatment cycles and therefore increased anticancer efficacy. The protein modification is straightforward with minimal perturbation of the tagged construct and can be applied to mAbs (fragments), other biologics, and nanoparticles. We expect that this technology will also find application as a companion imaging tool in biologics development and, specifically for the CA, in the controlled removal of, for example, antibody-drug conjugates or radiolabeled antibodies from blood. Finally, the presented components may allow organic chemistry to be conducted in humans.

\section{DISCLOSURE}

The costs of publication of this article were defrayed in part by the payment of page charges. Therefore, and solely to indicate this fact, this article is hereby marked "advertisement" in accordance with 18 USC section 1734 . No potential conflict of interest relevant to this article was reported.

\section{ACKNOWLEDGMENTS}

We thank Ebo Bos and Frans Kaspersen (former Organon) for insightful discussions, and we thank Ron Versteegen (SyMO-Chem), Sidney Wessels, Iris Verel, Monique Berben (Philips Research), Caren van Kammen, Carlijn van Helvert, and Melanie Blonk (Maastricht University) for support with chemical and in vivo experiments.

\section{REFERENCES}

1. Boswell CA, Brechbiel MW. Development of radioimmunotherapeutic and diagnostic antibodies: an inside-out view. Nucl Med Biol. 2007;34:757-778.

2. Sharkey RM, Goldenberg DM. Pretargeted radioimmunotherapy of cancer. In: Reilly RM, ed. Monoclonal Antibody and Peptide-Targeted Radiotherapy of Cancer. Hoboken, NJ: John Wiley and Sons; 2010:241-287.

3. Aweda TA, Beck HE, Wu AM, Wei LH, Weber WA, Meares CF. Rates and equilibria for probe capture by an antibody with infinite affinity. Bioconjug Chem. 2010;21:784-791.

4. Kuijpers WHA, Kaspersen FM, Veeneman GH, Van Boeckel CAA, Bos ES. Specific recognition of antibody-oligonucleotide conjugates by radiolabeled antisense nucleotides: a novel approach for two-step radioimmunotherapy of cancer. Bioconjug Chem. 1993;4:94-102.

5. Liu G, Mang'era K, Liu N, Gupta S, Rusckowski M, Hnatowich DJ. Tumor pretargeting in mice using ${ }^{99 \mathrm{~m}} \mathrm{Tc}-$ labeled morpholino, a DNA analog. $\mathrm{J} \mathrm{Nucl}$ Med. 2002;43:384-391.

6. Sletten EM, Bertozzi CR. Bioorthogonal chemistry: fishing for selectivity in a sea of functionality. Angew Chem Int Ed Engl. 2009;48:6974-6998.
7. Blackman ML, Royzen M, Fox JM. Tetrazine ligation: fast bioconjugation based on inverse-electron-demand Diels-Alder reactivity. J Am Chem Soc. 2008;130: 13518-13519.

8. Rossin R, Renart Verkerk P, van den Bosch S, et al. In vivo chemistry for pretargeted tumor imaging in live mice. Angew Chem Int Ed Engl. 2010;49: 3375-3378.

9. Devaraj NK, Thurber GM, Keliher EJ, Marinelli B, Weissleder R. Reactive polymer enables efficient in vivo bioorthogonal chemistry. Proc Natl Acad Sci USA. 2012;109:4762-4767.

10. Zeglis BM, Sevak KK, Reiner T, et al. A pretargeted PET imaging strategy based on bioorthogonal Diels-Alder click chemistry. J Nucl Med. 2013;54:1389-1396.

11. Breitz HB, Weiden PL, Beaumier PL, et al. Clinical optimization of pretargeted radioimmunotherapy with antibody-streptavidin conjugate and ${ }^{90}$ Y-DOTAbiotin. J Nucl Med. 2000;41:131-140.

12. Rossin R, Van den Bosch SM, ten Hoeve W, et al. Highly reactive transcyclooctene tags with improved stability for Diels-Alder chemistry in living systems. Bioconjug Chem. 2013;24:1210-1217.

13. Guide for the Care and Use of Laboratory Animals. Washington, DC: Government Printing Office; 1985. NIH publication 86-23.

14. Segars WP, Tsui BMW, Frey EC, Johnson GA, Berr SS. Development of a 4-D digital mouse phantom for molecular imaging research. Mol Imaging Biol. 2004;6:149-159.

15. Marshall D, Pedley RB, Melton RG, Boden JA, Boden R, Begent RH. Galactosylated streptavidin for improved clearance of biotinylated intact and $F\left(a^{\prime}\right) 2$ fragments of an anti-tumor antibody. Br J Cancer. 1995;71:18-24.

16. Alexis F, Pridgen E, Molnar LK, Farokhzad OC. Factors affecting the clearance and biodistribution of polymeric nanoparticles. Mol Pharm. 2008;5:505-515.

17. Patel S, Stein R, Ong GL, Goldenberg DM, Mattes MJ. Enhancement of tumorto-nontumor localization ratios by hepatocyte-directed blood clearance of antibodies labeled with certain residualizing radiolabels. J Nucl Med. 1999;40: 1392-1401.

18. Ogawara K, Yoshida M, Higaki K, et al. Hepatic uptake of polystyrene microspheres in rats: effect of particle size on intrahepatic distribution. $J$ Control Release. 1999;59:15-22.

19. Staud F, Nishikawa M, Takakura Y, Hashida M. Liver uptake and hepato-biliary transfer of galactosylated proteins in rats are determined by the extent of galactosylation. Biochim Biophys Acta. 1999;1427:183-192.

20. Axworthy DB, Reno JM, Hylarides MD, et al. Cure of human carcinoma xenografts by a single dose of pretargeted yttrium-90 with negligible toxicity. Proc Natl Acad Sci USA. 2000;97:1802-1807.

21. Lewis MR, Wang M, Axworthy DB, et al. In vivo evaluation of pretargeted ${ }^{64} \mathrm{Cu}$ for tumor imaging and therapy. J Nucl Med. 2003;44:1284-1292.

22. McBride WJ, Zanzonico P, Sharkey RM, et al. Bispecific antibody pretargeting PET (immunoPET) with an ${ }^{124}$ I-labeled hapten-peptide. J Nucl Med. 2006;47: 1678-1688.

23. Moosmayer D, Berndorff D, Chang C-H, et al. Bispecific antibody pretargeting of tumor neovasculature for improved systemic radiotherapy of solid tumors. Clin Cancer Res. 2006;12:5587-5595.

24. Mohsin H, Jia F, Sivaguru G, et al. Radiolanthanide-labeled monoclonal antibody CC49 for radioimmunotherapy of cancer: biological comparison of DOTA conjugates and ${ }^{149} \mathrm{Pm},{ }^{166} \mathrm{Ho}$, and ${ }^{177} \mathrm{Lu}$. Bioconjug Chem. 2006;17:485-492.

25. Graves SS, Dearstyne E, Lin Y, et al. Combination therapy with pretarget CC49 radioimmunotherapy and gemcitabine prolongs tumor doubling time in a murine xenograft model of colon cancer more effectively than either monotherapy. Clin Cancer Res. 2003;9:3712-3721.

26. Lewis MR, Zhang J, Jia F, et al. Biological comparison of ${ }^{149} \mathrm{Pm}-,{ }^{166} \mathrm{Ho}-$, and ${ }^{177} \mathrm{Lu}$-DOTA-biotin pretargeted by CC49 $\mathrm{scFv}$-streptavidin fusion protein in xenograft-bearing nude mice. Nucl Med Biol. 2004;31:213-223.

27. Pizzarello DJ, Witcofski RL. Medical Radiation Biology. 2nd ed. Philadelphia, PA: Lea and Febiger; 1982:151.

28. Chatal JF, Davodeau F, Cherel M, Barbet J. Different ways to improve the clinical effectiveness of radioimmunotherapy in solid tumors. J Cancer Res Ther. 2009;5(suppl):S36-S40.

29. Sharkey RM, Karacay H, Liywin S, et al. Improved therapeutic results by pretargeted radioimmunotherapy of non-Hodgkin's lymphoma with a new recombinant, trivalent, anti-CD20, bispecific antibody. Cancer Res. 2008;68:5282-5290. 